\title{
Letter to the Editor: Hybrid Prosthetic Treatment of the Edentulous Mandible with Two or Four Implants - A Literature Review
}

\author{
Srdjan Postic* \\ Faculty of Dental Medicine, University of Belgrade, Belgrade, Serbia
}

*Corresponding author: Srdjan Postic, Clinic of Dental Prosthetic, Faculty of Dental Medicine, University of Belgrade, Belgrade, Serbia, Tel: +381 11 2435 719/ext 455 ; +86-21-58386256; E-mail: srdjan.postic@stomf.bg.ac.rs

Rec Date: Mar 30, 2015; Acc Date: April 7, 2015; Pub Date: April 13, 2015

Copyright: ( 2015 Postic et al., This is an open-access article distributed under the terms of the Creative Commons Attribution License, which permits unrestricted use, distribution, and reproduction in any medium, provided the original author and source are credited.

\section{Letter to the Editor}

Dear Editor,

I have carefully read the manuscript entitled: "Hybrid prosthetic treatment of the edentulous mandible with two or four implants - a literature review "and noted that it is a review of the works. In this paper, the authors look at representations of situations where the implants were successfully placed in the lower jaw. The authors also propose a clear and meaningful use four instead of two implants in the lower or the upper edentulous jaw, which is a great quality in this manuscript of reviewed character.

Although it contains quite a sufficient number of isolated cases of clinical practice in which the implants are placed prosthesis work, in my opinion, has certain drawbacks that should have been annotated, or at least indicated.

Firstly, the work does not show situations where there is no local or systemic osteoporosis in the jaw. It was advocated in the literature that osteoporosis affects more than $50 \%$ of the human population after 50 years of age (where there is information that is in certain situations, osteoporosis and even earlier in the present human organism, are also present data from literature when osteoporosis is present due to lactation as a transient disorder);

In addition, the authors of the manuscript should have commented on the situation in which the implants were embedded in the jaw or patients with skeletal class II, or in the jaw in patients with skeletal class III.

Finally, the paper have not been described in detail the situation, that is not discussed in more detail situations when certain types of implants -as for example mini implants built as temporary, while at the same time takes oseontegracija implants that have built-placed for example in the lateral sector in the sense that remain permanently, not temporarily (a clinical situations when a patient in the lower jaw of his/her mouth has had inserted 4 permanent implant-in the process of oseointegration, and additional 4 mini implants for the retention of temporary provisional denture)

After reviewing manuscript entitled "Hybrid prosthetic treatment of the edentulous mandible with two or four implants - a literature review" for the short Dentistry Journal. I suggested revisions regarding barriers and indications for clinical use of two or four implants, as well as short paragraph indicating temporary uses of mini-implants.

For the foregoing reasons, I believe that the text and content of the work should be partially extended and amended, and only then plan the eventual printing work in a professional journal.

\section{Best regards,}

Srdjan Postic 